\title{
On the energy dependence of proton beam extraction with a bent crystal
}

\author{
G. Arduini, K. Elsener, G. Fidecaro, M. Gyr, \\ W. Herr, J. Klem, U. Mikkelsen†, E. Weisse \\ CERN, SL Division, CH-1211 Geneva 23
}

\begin{abstract}
Proton beam extraction from the CERN SPS by means of a bent silicon crystal is reported at three different energies, $14 \mathrm{GeV}, 120 \mathrm{GeV}$ and $270 \mathrm{GeV}$. The experimental results are compared to computer simulations which contain a sound model of the SPS accelerator as well as the channeling phenomena in bent crystals. The overall energy dependence of crystal assisted proton beam extraction is understood and provides the basis to discuss such a scheme for future accelerators.
\end{abstract}

to be submitted to Physics Letters B

Geneva, Switzerland

November 19, 1997

* Also: Helsinki Inst. of Physics, Finland

$\dagger$ Also: ISA, Aarhus University, Denmark 


\section{Introduction}

Proton extraction with a bent crystal depends on parameters that change with the beam energy. Beam diffusion and the resulting impact parameters and angles depend on the beam energy and on the excitation that is used to make the beam particles hit the crystal. When the particles enter the crystal, their interactions are energy dependent: multiple scattering, critical angle of channeling, and dechanneling in straight and bent parts of the crystal change as a function of beam energy. Consequently, the extraction efficiency for a given extraction set-up depends strongly on the energy of the particles. For an extrapolation of the results up to LHC energies, it is necessary to have a high level of confidence in the models and the test of these models is the prime purpose of this study. In the SPS we had the unique opportunity to extract protons at three beam energies: $14 \mathrm{GeV}, 120 \mathrm{GeV}$ and $270 \mathrm{GeV}$. For all energies a beam could be extracted with a good signal to background ratio and extraction efficiencies, extracted beam sizes and angular scan widths could be measured. The crystal properties such as length, thickness and bending radius were designed and originally optimized for an energy of $120 \mathrm{GeV}[1,2]$ and significant effects are expected for the large energy range available to us. Although extraction of protons has been demonstrated up to an energy of $900 \mathrm{GeV}[3]$, the experimental technique used was significantly different and a direct comparison is very difficult. Our study is therefore restricted to the experiments made at the three SPS energies where the setup and experimental procedure was identical for all experiments.

\section{Planar channeling and deflection in bent crystals}

Protons entering a crystal lattice with a small angle relative to a major planar direction can be 'channeled', i.e. trapped in the strong potential well created by the atoms in the crystal lattice [4]. The limiting angle for this process is the critical angle $\psi_{c}$ for planar channeling [5]. This angle scales as $1 / \sqrt{p}$ with the particle momentum $p$ and is given in Tab. 1 for the three energies used. Once channeled, protons remain trapped by the crystalline fields and travel through the crystal, even if it is slightly bent. Processes leading to losses of protons out of the channeling potential well are called 'dechanneling processes'. In straight crystals, these are mainly due to collisions with electrons in the crystal. In a bent crystal, additional dechanneling occurs due to the deformation of the channeling potential expressed by the bending dechanneling parameter $F$ [6]. Analytical models have been developed to describe the channeling and deflection processes. These models are found to be in good agreement with experiments using high energy proton beams deflected by silicon or germanium crystals $[7,8]$. The energy dependence of the dechanneling processes can be summarized as follows: The multiple scattering dechanneling length $L_{D}$ scales with $p$ and a 
shorter crystal would be an advantage at low energies. The number of particles remaining channeled follow the exponential law $N=N_{0} \exp \left(-l / L_{D}\right)$, where $l$ is the length of the crystal. The bending dechanneling follows a more complicated law and the dechanneling length $L_{D}$ has to be modified by the dechanneling parameter $F$ according to $L_{B}=L_{D} \cdot(1-F)^{2}$. The bending dechanneling $F$ is inversely proportional to the bending radius, i.e. depends on bending angle $\theta$ and length $l$, and is proportional to the momentum of the particle. This implies that longer crystals are favoured at higher energies. This is demonstrated in Tab. 1 where the dechanneling fraction and the dechanneling lengths are given for three energies and the standard crystal parameters as used in this experiment and given in the table. Also given are the probabilities $P_{r}$ that a channeled particle remains channeled in the crystal over its full length. This probability is calculated as:

$$
P_{r}=(1-F(\theta, l, p)) \cdot \exp \left(-l_{s} / L_{D}\right) \cdot \exp \left(-l_{b} / L_{B}\right)
$$

where $l_{s}$ and $l_{b}$ are the lengths of the straight and bent parts of the crystal. The energy dependence is apparent and in particular at $14 \mathrm{GeV}$ the major fraction of the channeled particles will be lost due to multiple scattering dechanneling. A much shorter crystal would be required for an extraction at $14 \mathrm{GeV}$ with a good efficiency. At $270 \mathrm{GeV}$ the normal dechanneling process is negligible but the dechanneling fraction $F$ for a $4 \mathrm{~cm}$ long crystal bent by $8.5 \mathrm{mrad}$ becomes the dominant factor. Summarizing, for a given deflection angle, the deflection of protons in a bent crystal can be performed with an optimum efficiency, if the correct crystal length is chosen for the beam energy in question. Given the energy and the bending angle, the above equation can be used to get the optimum crystal length for deflection [7].

\section{Beam extraction by the single- or multi-pass process}

The impact parameter of a particle incident on a crystal is usually very small and the quality of the surface becomes important. For a perfect crystal the surface is parallel to the crystalline planes. In practice, the crystals cannot be cut exactly parallel and the roughness of the surface may be larger than the impact parameter. As a consequence, a certain width of the surface is inefficient for channeling particles. When a proton passes the crystal in this inefficient region or is dechanneled, it experiences multiple scattering and is deflected by a random angle $\theta_{m s}$ with a nearly Gaussian distribution with zero mean value and a width [9]:

$$
\theta_{m s}=\frac{13.6 \mathrm{MeV}}{\beta_{r} c p} \sqrt{\frac{L_{e f f}}{X_{0}}}\left(1+0.038 \ln \left(\frac{L_{e f f}}{X_{0}}\right)\right)
$$

where $c p$ is the momentum, $\beta_{r} \sim 1$, and $X_{0}$ is the radiation length of the crystal ( $\sim 9.4 \mathrm{~cm}$ for silicon). For a particle traversing the full crystal length of $4 \mathrm{~cm}$ 
this angle $\theta_{m s}$ is approximately $71.5 \mu \mathrm{rad}$ at $120 \mathrm{GeV}$. Due to the small impact parameters and because the crystal is bent away from the beam axis, the particles which undergo multiple scattering do not cross the full crystal length but only a small part of it (i.e. $L_{e f f}$, c.f. $[2,10]$ ). The multiple scattering determines the impact parameters for further intercepts on the crystal [2]: strong scattering leads to large impact parameters. For initial impact parameters smaller than the inefficient surface layer, multi-pass extraction is the only possible process and the typical impact parameters expected in our experiments are usually in the submicron level or smaller. It has been shown [11] that the multi-pass extraction process is the dominant factor for extraction from the SPS, i.e. particles traverse the crystal multiple times before they are eventually channeled. The initial impact parameters and angles, i.e. for the first encounter of a particle with the crystal, are less important for a multi-pass process and their energy dependence can be neglected. However the beam optics of the accelerator at the position of the crystal such as horizontal and vertical $\beta$-function plays an important role and determines the effect of the scattering process on further turns [12]. For a well chosen extraction optics and assuming a dominant contribution from the multi-pass process, the energy dependence of the overall extraction efficiency is largely determined by dechanneling processes. When the crystal is bent in the horizontal plane, the horizontal motion is confined within the vertical crystalline planes and the horizontal profiles are determined by the geometrical acceptance of the crystal and the material between the crystal and the detector system: at the exit of the crystal the extracted beam cannot be wider than the thickness of the crystal and its divergence must be smaller than twice the critical angle. In the vertical direction the particles are not confined by the channeling potential and it is expected that the width of the vertical profiles of channeled particles is increased due to multiple scattering, in particular for the lower beam energies.

\section{Extraction experiments at the SPS}

Proton beam extraction has been demonstrated repeatedly at the CERN SPS [1, 2]. These experiments were designed to study extraction of protons at an energy of $120 \mathrm{GeV}$. For the desired bending angle of $8.5 \mathrm{mrad}$, the crystal parameters such as length and thickness were originally optimized for this energy. We have chosen crystals 30 and $40 \mathrm{~mm}$ long and $1.5 \mathrm{~mm}$ thick. Details about the crystals used and their properties can be found in $[2,13]$. The experimental layout is described in detail in [2] and schematically shown in Fig. 1. Protons from a circulating beam are diffusing towards a bent silicon crystal. If their impact angle and position are favourable, they may be channeled and extracted towards a set of detectors. The crystalline planes have to be aligned with the beam and therefore the crystal is mounted on a remote controlled goniometer. In order to find the optimum crystal angle for a given accelerator setting, the goniometer is 
turned in small steps and the extracted beam rate observed as a coincidence of three scintillation counters. Such 'angular scans' are performed repeatedly during the experiment. Once the optimum alignment is found, the extracted beam is recorded in a scintillation counter hodoscope, consisting of 32 horizontal and 32 vertical strips of $1 \mathrm{~mm}$ width [2]. This allows the detailed analysis of beam profile width and gives an accurate (background subtracted) extracted beam intensity. When all losses are assumed to be due to the presence of the crystal, which was verified experimentally, the extraction efficiency is defined as the ratio between beam extracted and beam lost $\epsilon_{\text {extr }}=I_{\text {extr }} / I_{\text {lost }}$. This efficiency can be separated into two parts, the probability for a proton to be captured initially $\left(P_{c h}\right)$ and the probability to remain channeled $P_{r}$ (eq. (1)). The energy dependence of $P_{c h}$ is mainly due to the changed critical angle which scales as $1 / \sqrt{p}$ which reduces the geometrical acceptance of the crystal at higher energies. The horizontal divergence of the beam grows faster at lower energies and both effects largely compensate each other [13] leading to a weak energy dependence. For a multipass extraction the number of passes through the crystal plays an important role and more passes and hence more chances to get channeled and extracted are possible when the multiple scattering is weaker for higher beam energies.

\section{Computer simulations}

In this report we describe only the very general structure of the simulation program and details can be found in $[10,13]$. Other simulation programs have been developed [14] and give similar results. Our simulation is split into two independent modules: the simulation of beam diffusion and the simulation of the interaction with the crystal and the multi-pass process. In the first part the motion of the particles is simulated under the influence of a diffusion mechanism (here transverse noise [15]) that makes the particles diffuse outwards in the horizontal plane until they hit the crystal. The initial impact parameters and angles are recorded. The parameters for the diffusion mechanism (e.g. noise amplitude) can be varied and their effect on the impact parameter and angle distributions was studied. In the second part, the impact parameters and impact angles obtained from the diffusion module are used as initial coordinates for particles that interact with the crystal and are tracked around the accelerator until they are extracted or lost. The interaction with the crystal is described in [10, 13]. By changing crystal or accelerator parameters their effect on the multi-pass process can be studied. The simulation program can reproduce the extracted beam profiles and the extraction efficiences when the number of extracted particles is compared to the number of protons initially hitting the crystal. An angular scan can be simulated and the profiles and efficiences during a scan are obtained. 


\section{Results}

In order to study the energy dependence of the extraction with a crystal, experiments were made at three energies: 14, 120 and $270 \mathrm{GeV}$, covering an energy range as wide as possible at the SPS. At all three energies we have extracted protons with a good signal to background ratio and angular scans, extracted beam profiles and extraction efficiencies were measured. Details of the analysis can be found in [13]. It has already been argued, that the energy dependence of the interaction of protons with the crystal and the accelerator should result in differences in the measurable quantities such as efficiency, extracted beam sizes and angular scans. We shall therefore compare the measurements obtained at the three energies and the results from the simulation. This provides a good test of the models used for the extraction and channeling processes.

Angular scans are made to align the crystalline planes with the incoming beam. During a scan the extracted beam rate is recorded and the horizontal and vertical profiles of the extracted beam are measured with the scintillation counter hodoscope. The extracted beam rate is largest when the alignment is optimum. The width of an angular scan is determined by the size of the critical angle, the divergence of the incoming beam and the multi-pass behaviour of the extracted particles and should therefore exhibit an energy dependence. It was also found [2] that the vertical profiles change their widths as a function of the alignment angle, i.e. they are narrowest when the crystal is well aligned with the beam. This is shown in Fig. 2 where an angular scan at $270 \mathrm{GeV}$ is shown together with the measured vertical profile width. A clear correlation between the alignment and the profile width is shown. This behaviour can be understood by considering the number of passes in the crystal that are necessary for the protons to be channeled and extracted. At each pass through the crystal that does not lead to extraction, the angular distribution of the protons gets wider in both horizontal and vertical planes due to multiple scattering in the crystal material. With a well aligned crystal, fewer passes are needed and the width of the vertical profile suffers less from multiple scattering. This is demonstrated in Fig. 3 where the vertical profile width is shown for a simulated angular scan together with the number of traversals through the crystal before the particle was channeled. The widths of the scans at the three energies depend on the exact experimental conditions. For a single pass extraction one would expect a width comparable to twice the critical angle and therefore a strong energy dependence. At all energies, the narrowest scans were measured around $70-80 \mu \mathrm{rad}$ up to values of $150 \mu \mathrm{rad}$ and no sign of single pass extraction was found in any of the experiments. The horizontal and vertical profiles of the extracted beams are shown for 14 and $270 \mathrm{GeV}$ in Figs. 4 and 5 together with the profiles obtained from the simulation. The qualitative agreement between the experimental results and the simulation is very good. For a quantitative comparison, the measured and simulated r.m.s. widths for the horizontal and vertical extracted beam profiles at the three beam 
energies are shown in Tab. 2. The background in the measured profiles has been subtracted. An excellent agreement between the measured and simulated profile widths is found, indicating that the model is correct at the energies investigated.

An absolute prediction of the extraction efficiency proved to be very difficult $[2,13]$ as it strongly depends on some parameters that are less well known, e.g. the width of the inefficient layer and the exact distributions of impact parameter and angle. The prediction can vary by up to a factor of two, depending on the assumptions. It is mainly the capture probability $P_{c h}$ that is affected by these uncertainties and less the dechanneling probability. The relative efficiences should show the expected behaviour as a function of the beam energy. The capture probability also depends on the accelerator parameters (e.g. drift speed and beam divergence) and small changes to the conditions can lead to different measured efficiencies. To minimize this variation in the comparison, we give the highest measured extraction efficiencies at the three beam energies in Tab. 3 and compare them to the efficiencies obtained from simulations. To show the energy dependence, the simulated values shown in Tab. 3 are normalized to the measured values at $120 \mathrm{GeV}$. A very good agreement is found for the measured and simulated energy dependence. The efficiency at $14 \mathrm{GeV}$ is much lower due to multiple scattering and dechanneling. Due to large multiple scattering angles at low energy, the divergence of the incident beam grows fast during multiple passes through the crystal. Furthermore, on the average the particles make fewer passes through the crystal and therefore have fewer chances to be extracted. The dechanneling length at $14 \mathrm{GeV}$ is much shorter (Tab. 1) than at higher energies, which as well reduces the deflection and extraction efficiencies by a large factor. For a quantitative comparison we have given the probability that a channeled particle remains channeled. Although this probability is larger for $120 \mathrm{GeV}$ protons, the extraction efficiency at $270 \mathrm{GeV}$ is slightly larger due to the increased number of passes through the crystal. The simulation shows that the average number of passes through the crystal increases from around 3 to 5 , depending on the exact conditions. The increase of the horizontal beam divergence due to the multiple scattering is also smaller and both effects increase the capture probability $P_{c h}$, compensating for the smaller geometrical acceptance due to the decreased critical angle. The relative extraction efficiencies are very well reproduced by the models used in the simulation. We conclude that the basic mechanisms and processes are well understood and allow a prediction towards higher energies.

\section{Consequences for the $\mathrm{LHC}$}

It was mentioned that the correct choice of the crystal length is important to obtain a high extraction efficiency. Having confidence in the models, we attempt such an optimization for the suggested LHC parameters. We have not tried to 
fully simulate an extraction process for the LHC. Instead we use plausibility arguments and the assumption that the extraction relies only on the multi-pass process. Therefore we do not have to speculate about possible diffusion mechanisms and the resulting impact parameters. A more complete simulation was done by V. Biryukov [14] and the conclusions are very similar. The multiple scattering dechanneling length for $\mathrm{Si}(110)$ at $7 \mathrm{TeV}$ is $2.91 \mathrm{~m}$ and therefore plays only a minor role for dechanneling effects. The main contribution to dechanneling of captured protons is due to bending dechanneling. Rather small bending angles and large crystal length are therefore desired. Equation (1) can be used to find the length $l$ for which the probability $P_{r}$ has a maximum. For the LHC the optimum length can become too large and impractical, in particular for larger bending angles. Two bending angles are envisaged for the LHC, 0.7 and $1.0 \mathrm{mrad}$. For both bending angles the maximum of the probability $P_{r}$ is around $70 \%$ for a length of 20 to $30 \mathrm{~cm}$ but lengths above $10 \mathrm{~cm}$ give already a probability of approximately $60 \%$. For the more conservative value of only $0.7 \mathrm{mrad}$ bending angle, a crystal length of $5 \mathrm{~cm}$ gives a probability around $50 \%$. Such a combination is most promising because the simulations [13, 14] suggest shorter crystals when multi-pass extraction is a dominant process.

\section{Conclusions}

In order to study the energy dependence of proton extraction with a bent silicon crystal, we have made experiments at three different beam energies at the CERN SPS: 14, 120 and $270 \mathrm{GeV}$. At all energies extracted beam was observed with a good signal to background ratio which allowed to measure the relevant parameters such as width of extracted beam profiles and extraction efficiencies as a function of the beam energy. Assuming a dominant contribution from the multi-pass extraction process, the main contribution to the energy dependence of the extraction efficiency comes from the interaction with the crystal, i.e. multiple scattering and dechanneling. The models used describe the observations very well and allow an extrapolation towards LHC energies.

\section{Acknowledgements}

We wish to thank all the members of the former RD22 collaboration, in particular S.P. Møller and E. Uggerhøj, for their continued interest and support of these experiments. We thank A. Freund, R. Hustache and A. Paul of ESRF Grenoble for the careful cutting, polishing and bending of the crystals. We wish to thank the SPS operations team, in particular X. Altuna and A. Ferrari, for the assistance during the preparation and running of the experiments. The help of G. Ferioli and the beam instrumentation group is greatfully acknowledged. 


\section{References}

[1] H. Akbari et al., Phys. Lett. B313 (1993) 31.

[2] K. Elsener et al., Nucl. Instr. Meth. B119, (1996) 215.

[3] T. Murphy et al., Nucl.Instr.Meth. B119, (1996) 231.

[4] H.H. Andersen et al. (eds.), Nucl. Instr. Meth. B119, Nos 1,2 (1996).

[5] J. Lindhard, Phys. Lett. 12, (1964) 126.

[6] J.A. Ellison et al., Nucl. Phys. B206 (1982) 205. H. Kudo, Nucl. Inst. and Meth. 189 (1981) 609.

[7] U. Mikkelsen, PhD thesis, University of Aarhus, Denmark, (1997) (unpublished).

[8] C. Biino et al., Phys. Lett. B403, (1997) 163.

[9] Particle Data Group, Phys. Rev. D 54, (1996) part I.

[10] G. Vuagnin, CERN/SL/Note 95-24 (AP) (unpublished).

[11] X. Altuna et al., Phys.Lett. B357, (1995) 671.

[12] S. Bardin, CERN/SL/Note 92-52 (AP) (unpublished).

[13] J. Klem, PhD Thesis, Helsinki Univ. of Technology, Finland, (1997) (unpublished).

[14] V.M. Biryukov, Phys. Rev. Lett. Vol. 74, Number 13, (1995) 2471.

[15] W. Herr, CERN/SL 92-53 (AP) (unpublished). 


\section{Tables}

Table 1: Critical angles and dechanneling parameters for 14, 120 and $270 \mathrm{GeV}$ for protons in Si (110). Calculated for a crystal of $4 \mathrm{~cm}$ length $(3 \mathrm{~cm}$ bent, $1 \mathrm{~cm}$ straight) and a deflection angle of $8.5 \mathrm{mrad}$.

\begin{tabular}{cccccc}
\hline Energy E $[\mathrm{GeV}]$ & $\psi_{c}[\mu \mathrm{rad}]$ & $L_{D}[\mathrm{~cm}]$ & $F$ & $L_{B}[\mathrm{~cm}]$ & $P_{r}$ \\
\hline 14 & 42.3 & 0.92 & 0.02 & 0.89 & 0.011 \\
120 & 14.4 & 6.60 & 0.18 & 4.45 & 0.360 \\
270 & 9.6 & 13.90 & 0.39 & 5.22 & 0.320 \\
\hline
\end{tabular}

Table 2: Measured and simulated RMS widths for the horizontal and vertical extracted beam profiles.

\begin{tabular}{ccccc}
\hline $\begin{array}{c}\text { SPS beam } \\
\text { energy } \\
{[\mathrm{GeV}]}\end{array}$ & $\begin{array}{c}\text { Measured } \\
\text { horiz. width } \\
{[\mathrm{mm}]}\end{array}$ & $\begin{array}{c}\text { Simulated } \\
\text { horiz. width } \\
{[\mathrm{mm}]}\end{array}$ & $\begin{array}{c}\text { Measured } \\
\text { vert. width } \\
{[\mathrm{mm}]}\end{array}$ & $\begin{array}{c}\text { Simulated } \\
\text { vert. width } \\
{[\mathrm{mm}]}\end{array}$ \\
\hline 14 & $5.4 \pm 0.2$ & $4.2 \pm 0.1$ & $6.3 \pm 0.3$ & $6.0 \pm 0.2$ \\
120 & $0.7 \pm 0.2$ & $0.7 \pm 0.1$ & $2.6 \pm 0.3$ & $2.4 \pm 0.2$ \\
270 & $0.5 \pm 0.2$ & $0.5 \pm 0.1$ & $1.8 \pm 0.3$ & $1.6 \pm 0.2$ \\
\hline
\end{tabular}

Table 3: Measured and simulated extraction efficiencies at three different beam energies. The simulated efficiencies are normalized to match the experimental value at $120 \mathrm{GeV}$. The probability to remain channeled is given.

\begin{tabular}{cccc}
\hline $\begin{array}{c}\text { SPS beam } \\
\text { energy [GeV] }\end{array}$ & $\begin{array}{c}\text { Extraction } \\
\text { efficiency [\%] }\end{array}$ & $\begin{array}{c}\text { Simulated extr. } \\
\text { efficiency }[\%]\end{array}$ & $\begin{array}{c}P_{r} \\
{[\%]}\end{array}$ \\
\hline 14 & $0.55 \pm 0.3$ & $0.48^{*}$ & 1.10 \\
120 & $15.4 \pm 2.2$ & $15.4^{*}$ & 36.0 \\
270 & $18.6 \pm 2.7$ & $18.0^{*}$ & 32.0 \\
\hline
\end{tabular}




\section{Figures}

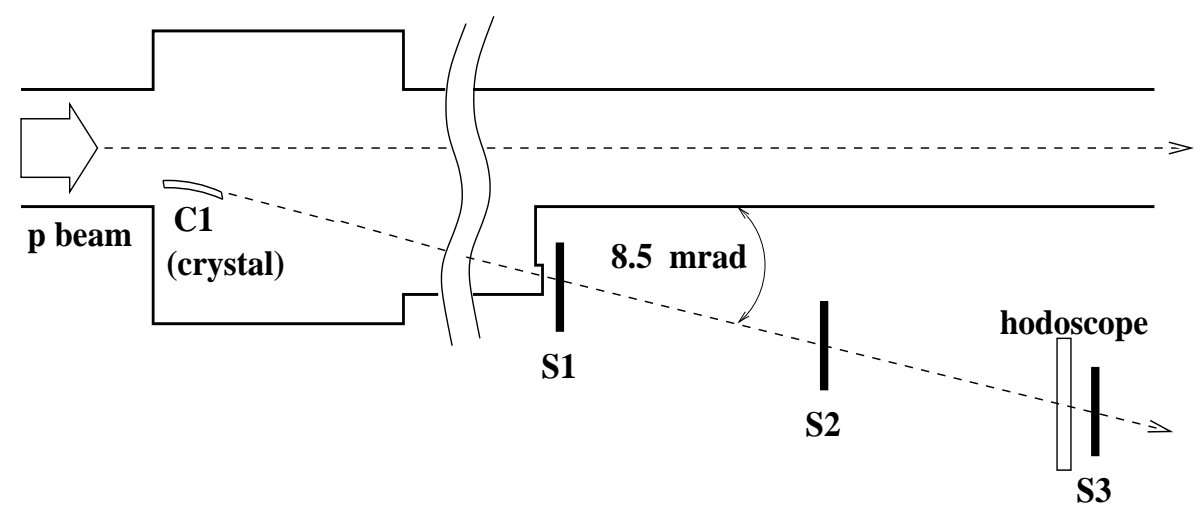

Figure 1: Schematic layout of the experiment at the SPS. 

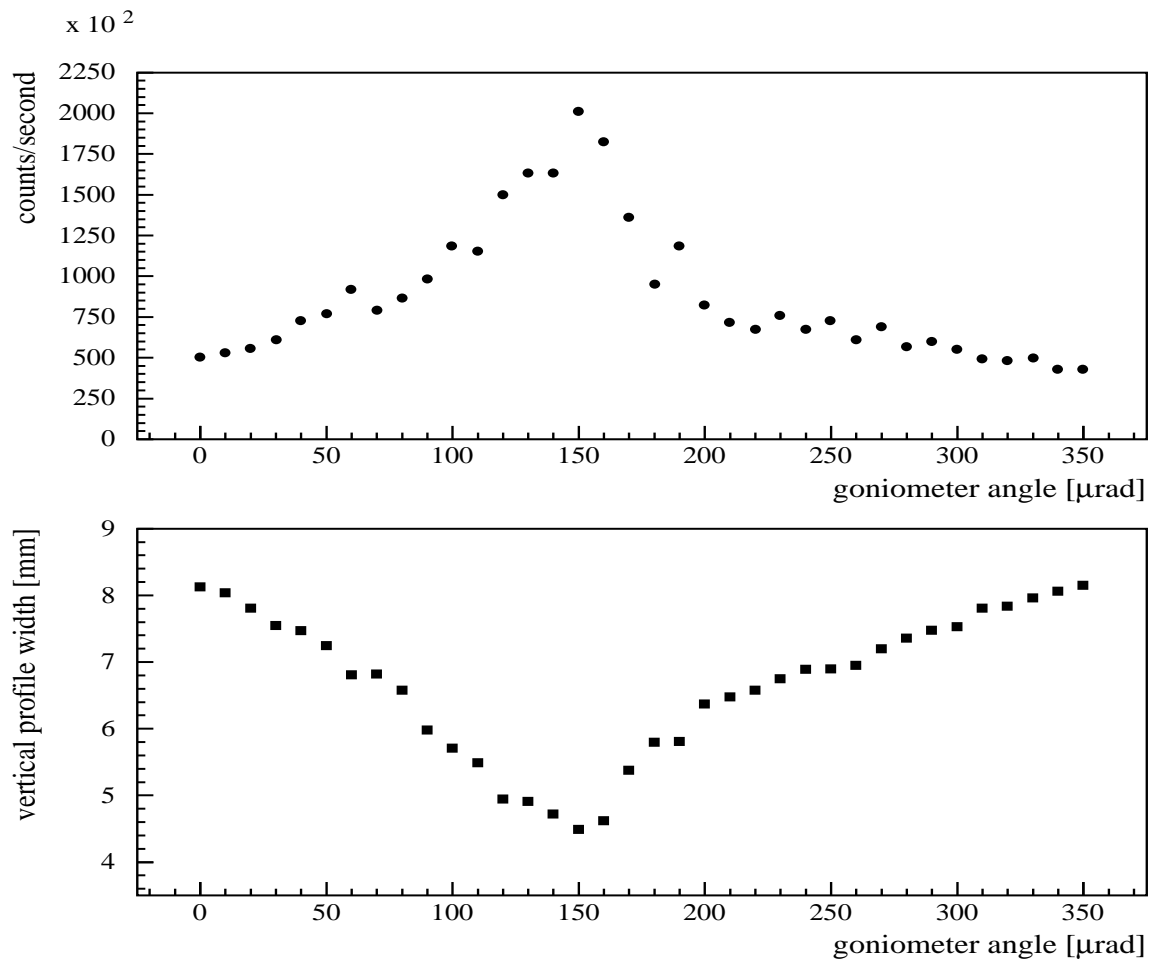

Figure 2: Angular scan and vertical profile width measured at $270 \mathrm{GeV}$.
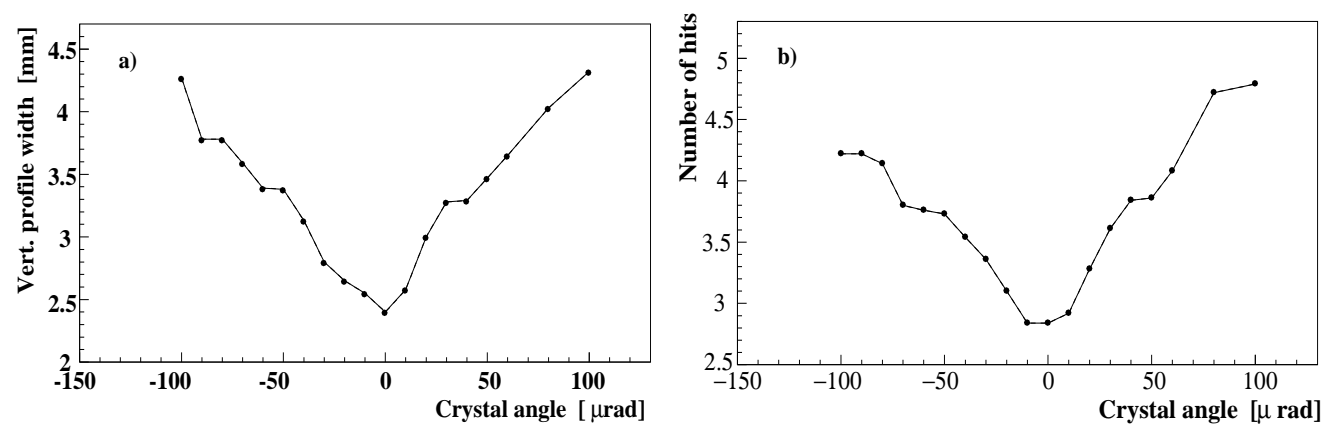

Figure 3: Vertical profile width simulated during angular scan (a) and number of passes through the crystal before channeling (b). 

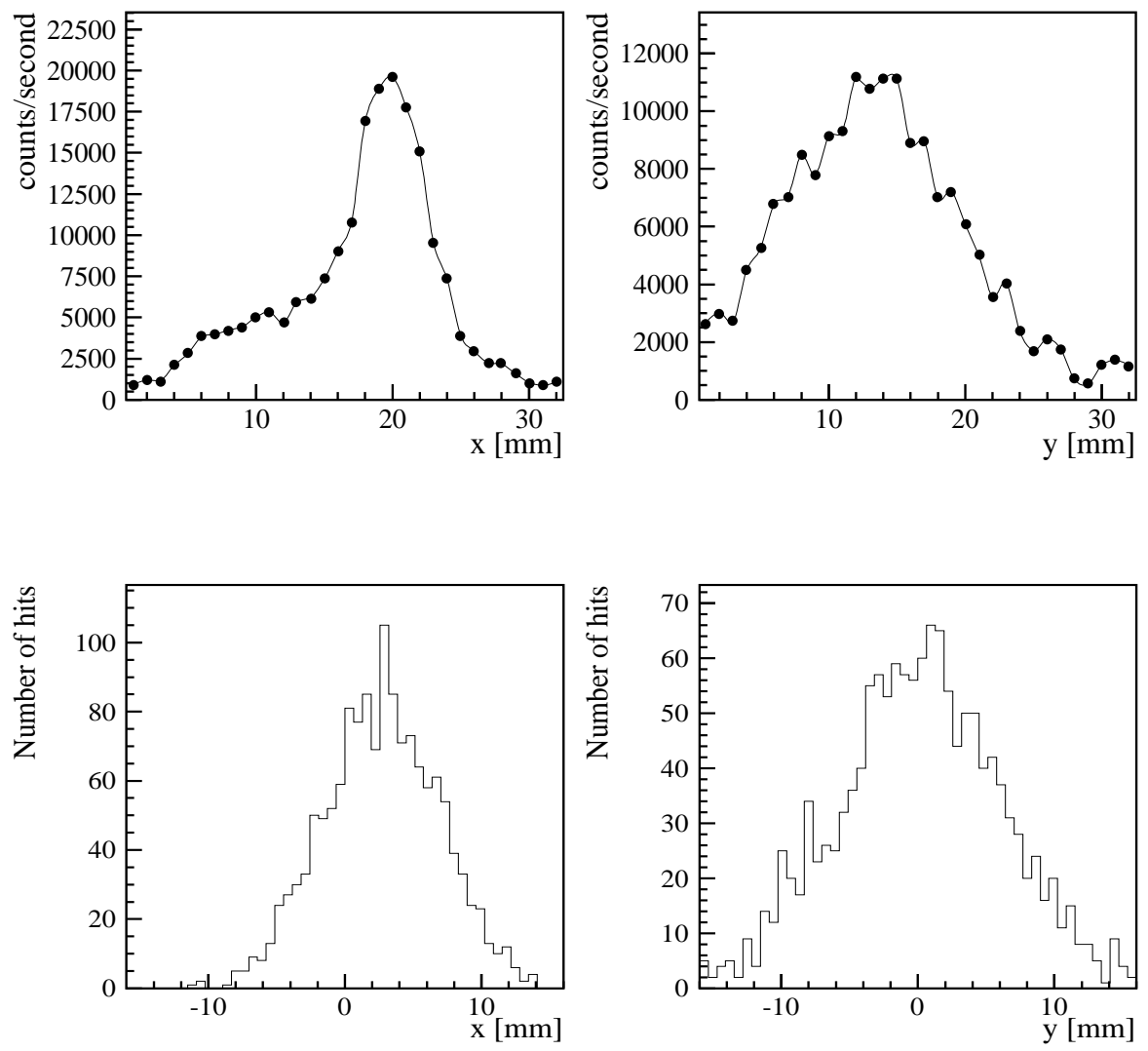

Figure 4: Proton beam extracted at $14 \mathrm{GeV}$. Horizontal and vertical profiles as measured by the hodoscope (top) and as obtained from the computer simulations (bottom). 

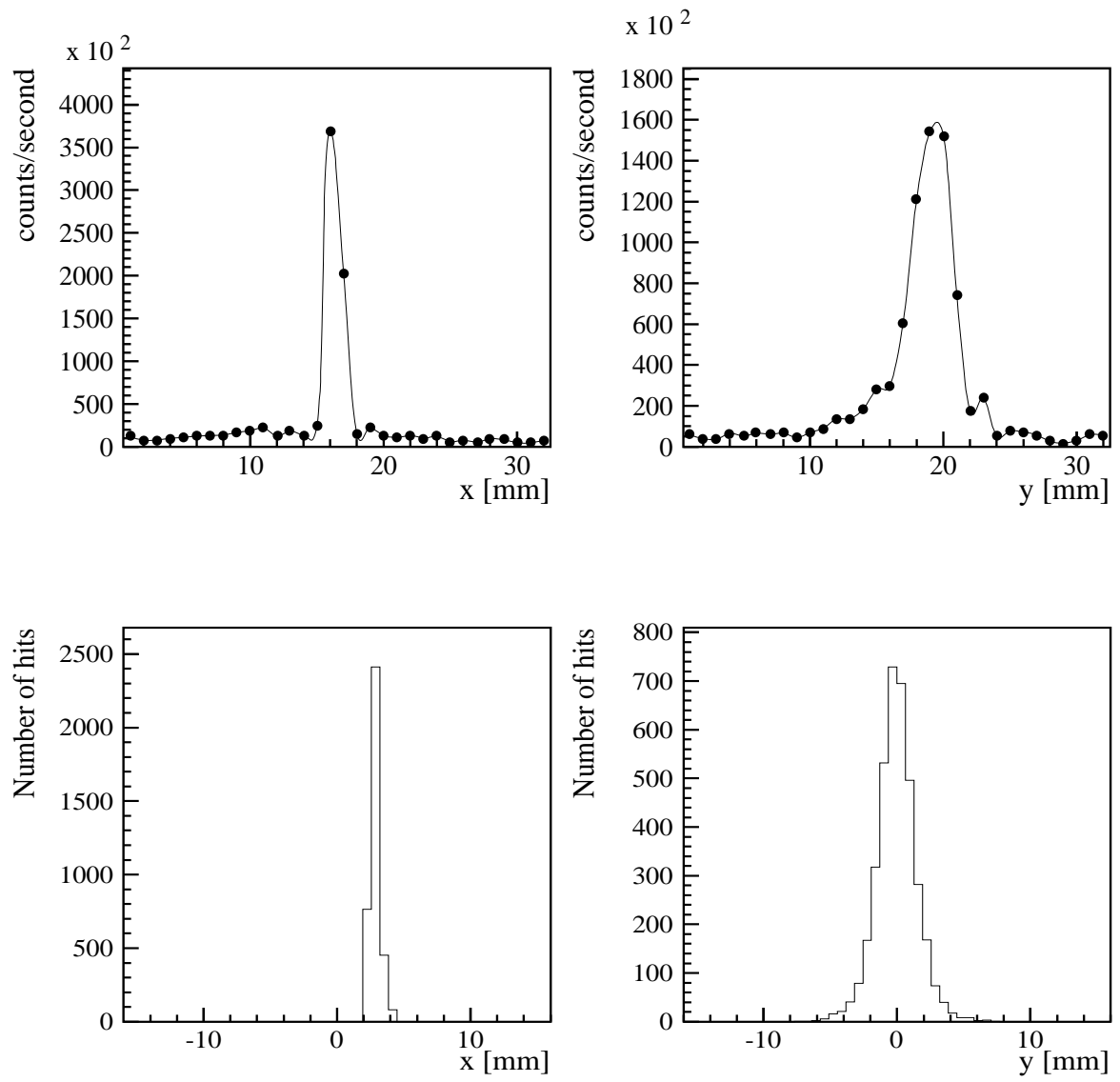

Figure 5: Proton beam extracted at $270 \mathrm{GeV}$. Horizontal and vertical profiles as measured by the hodoscope (top) and as obtained from the computer simulations (bottom). 